\section{International Scientific Journal Theoretical \& Applied Science}

p-ISSN: 2308-4944 (print) e-ISSN: 2409-0085 (online)

Year: $2014 \quad$ Issue: 12 Volume: 20

Published: $30.12 .2014 \quad$ http://www.T-Science.org
Vitaliy Anatolyevich Grinchenko $\mathrm{PhD}$ in Engineering Science, Senior lecturer Chair of Use of Electric Energy in Agriculture Stavropol State Agrarian University, Russia grinchen_ko@mail.ru

SECTION 23. Agriculture. Agronomy. The technique.

\title{
PROCESSES PRESSURE VARIATIONS IN THE A MILKING MACHINE
}

Abstract: The paper proposes a method for determining the duration of the processes of expiration and inleakage of air into the chamber milking cup during milking animals. Derived regularities allow obtaining the optimal design parameters of the pulsator milking machine with controlled duration transients.

Key words: milking machine, a vacuum between the walls of the chamber, milking cup, valve, pulsator, transition vacuum.

Language: Russian

Citation: Grinchenko VA (2014) PROCESSES PRESSURE VARIATIONS IN THE A MILKING MACHINE. ISJ Theoretical \& Applied Science $12 \quad$ (20): 86-89. http://dx.doi.org/10.15863/TAS.2014.12.20.18

\section{ПРОЦЕССЫ ИЗМЕНЕНИЯ ДАВЛЕНИЯ В ДОИЛЬНОМ АППАРАТЕ}

Аннотация: В статье предлагается метод определения длительности процессов истечения и натекания воздуха в межстенной камере доильного стакана во время доения животных. Выведенные закономерности позволяют получить оптимальные конструктивные параметры пульсатора доильного аппарата с управляемой длительностью переходньх процессов.

Ключевые слова: доильный аппарат, вакуум, межстенная камера, доильный стакан, клапан, пульсатор, переходный проиесс.

Истечение и натекание воздуха в межстенной камере доильного стакана влияет на деформацию сосковой резины, которая изменяет объем камер доильного стакана при доении. Эти процессы можно описать как истечение и натекание воздуха в камеры большого объема с постоянным давлением $[1,2]$ :

$$
\frac{P_{A T M}}{P_{i}} d\left(\frac{P_{i}}{P_{A T M}}\right)=-\frac{\mu \varphi S_{O}\left(S_{3}\right)}{V_{i}} \sqrt{P_{A T M} v_{A T M}} d t-\frac{d V_{i}}{V_{i}},
$$

$P_{\text {ATM }}$ - атмосферное давление;

$P_{i}$ - переменное давление в межстенных камерах;

$\mu$ - коэффициент расхода воздуха;

$\varphi-$ функция расхода воздуха;

$S_{O}\left(S_{3}\right)$ - площадь сечения линии истечения (натекания) воздуха;

$V_{i}$ - объем межстенной камеры и трубок доильного аппарата;

$v$ Aтм - удельный объем воздуха при атмосферном давлении.

Процессы движения воздуха в доильном аппарате считаются изотермическими, поскольку температура окружающей среды и температура воздуха в доильном аппарате отличается незначительно, а время процессов истечения и натекания сравнительно велико. Коэффициент расхода $\mu$ воздуха при истечении или натекании воздуха определяется выражением:

$$
\mu=\frac{1}{\sqrt{N\left(\lambda \frac{l_{T}}{D_{T}}+\varepsilon_{C}\right)}}
$$


Функция расхода воздуха $\varphi$ при изотермическом течении газа, которое имеет место в доильном аппарате, будет равна [3]:

$$
\varphi=\sqrt{\frac{1-\left(\frac{P_{i}}{P_{A T M}}\right)^{2}}{\varepsilon_{K M}-2 \ln \left(\frac{P_{i}}{P_{A T M}}\right)}},
$$

где $\varepsilon_{K M}-$ коэффициент сопротивления клапанного механизма пульсатора.

При нормальных условиях $v_{A T M}$ $=0,773 \mathrm{~m}^{3} / \kappa 2[4]$.

В межстенных камерах двухтактных доильных аппаратах объем системы истечения изменяется в пределах от $V$ до $V_{0}$ пропорционально отношению давлений $P_{i} / P_{A T M}$ [1], то есть:

$$
V_{i}=V+\left(V_{0}-V\right) \frac{P_{i}}{P_{A T M}}
$$

Продифференцировав (4), получим:

$$
d V_{i}=\left(V_{0}-V\right) d\left(\frac{P_{i}}{P_{A T M}}\right)
$$

Истечение воздуха из межстенной камеры происходит через отверстие в перегородке пульсатора площадью $S_{O}$ поперечного сечения. Поэтому уравнение, которое описывает истечение воздуха из межстенной камеры примет вид [5]:

$$
\frac{V d\left(\frac{P_{i}}{P_{A T M}}\right)}{\frac{P_{i}}{P_{A T M}}}+2\left(V_{0}-V\right) d\left(\frac{P_{i}}{P_{A T M}}\right)=-\mu \varphi S_{O} \sqrt{P_{A T M} v_{A T M}} d t .
$$

Решив (6), получим:

$$
V \ln \left(\frac{P_{i}}{P_{A T M}}\right)+2\left(V_{0}-V\right)\left(1-\frac{P_{i}}{P_{A T M}}\right)=\mu \varphi S_{O} \sqrt{P_{A T M} v_{A T M} t}
$$

Тогда продолжительность истечения воздуха из межстенной камеры равна:

$$
t_{a}=\frac{V \ln \left(\frac{P_{i}}{P_{A T M}}\right)+2\left(V_{0}-V\right)\left(1-\frac{P_{i}}{P_{A T M}}\right)}{\mu \varphi S_{O} \sqrt{P_{A T M} v_{A T M}}} .
$$

Выражение (8) описывает длительность процесса истечения воздуха и демонстрирует, что этот процесс зависит от объема межстенной камеры, габаритов системы истечения и площади отверстия, через которое происходит истечение воздуха [6].

При натекании воздуха площадь поперечного сечения атмосферного канала, через который в межстенную камеру поступает атмосферный воздух, постепенно увеличивается, изменяясь от нуля до $S_{3}$ мАХ при $X_{1} \rightarrow M A X$. Это происходит за промежуток времи $t_{C}$ [7]. Поэтому уравнение, описывающее процесс натекания воздуха в межстенную камеру доильных стаканов усовершенствованного доильного аппарата, примет вид $[8,9]$ :

$$
\frac{P_{A T M}}{P_{i}} d\left(\frac{P_{i}}{P_{A T M}}\right)=\frac{\mu \varphi S_{3} \sqrt{P_{A T M} v_{A T M}} d t}{\frac{P_{i}}{P_{A T M}}\left(V+\left(V_{0}-V\right) \frac{P_{i}}{P_{A T M}}\right)}-\frac{\left(V_{0}-V\right) d\left(\frac{P_{i}}{P_{A T M}}\right)}{V+\left(V_{0}-V\right) \frac{P_{i}}{P_{A T M}}} .
$$

Упростив (9), получим: 


$$
V d\left(\frac{P_{i}}{P_{A T M}}\right)+2\left(V_{0}-V\right) \frac{P_{i}}{P_{A T M}} d\left(\frac{P_{i}}{P_{A T M}}\right)=\mu \varphi S_{3} \sqrt{P_{A T M} v_{A T M}} d t
$$

Решив (10), получим:

$$
\begin{aligned}
& V\left(\left(\frac{P_{i}}{P_{A T M}}\right)_{M A X}-\frac{P_{i}}{P_{A T M}}\right)+\left(V_{0}-V\right)\left(\left(\frac{P_{i}}{P_{A T M}}\right)_{M A X}^{2}-\left(\frac{P_{i}}{P_{A T M}}\right)^{2}\right)= \\
& =\frac{\pi \mu \varphi \sqrt{P_{A T M} v_{A T M} t}}{8 \sqrt{3} X_{1}}\left[\frac{1}{\sqrt{3}}\left[\left(\left(D_{A}-\frac{X_{I}}{2 \sqrt{3}}\right)^{2}+\left(\frac{X_{I}}{2}\right)^{2}\right]^{\frac{3}{2}}-D_{A}^{3}\right)+\right. \\
& +\frac{15 D_{A}}{8 X_{1}}\left(\frac{2 X_{I}^{2}}{3}-\frac{D_{A} X_{I}}{\sqrt{3}}\right) \sqrt{\left(D_{A}-\frac{X_{I}}{2 \sqrt{3}}\right)^{2}+\left(\frac{X_{I}}{2}\right)^{2}}+\frac{5 \sqrt{3} D_{A}^{3}}{8}+ \\
& \left.+\frac{15 \sqrt{3} D_{A}^{3}}{16} \ln t\left(\ln \left[1-\frac{D_{A} \sqrt{3}}{2 X_{I}}+\frac{\sqrt{3}}{X_{I}} \sqrt{\left(D_{A}-\frac{X_{I}}{2 \sqrt{3}}\right)^{2}+\left(\frac{X_{I}}{2}\right)^{2}}\right]-\ln \frac{D_{A} \sqrt{3}}{2 X_{I}}\right)\right] .
\end{aligned}
$$

Учитывая взаимосвязь диаметра $D_{A}$ атмосферного канала с перемещением $X_{1}$ клапана пульсатора, получим:

$$
\begin{aligned}
& \left(\left(\frac{P_{i}}{P_{A T M}}\right)_{M A X}-\frac{P_{i}}{P_{A T M}}\right)\left[V+\left(V_{0}-V\right)\left(\left(\frac{P_{i}}{P_{A T M}}\right)_{M A X}+\frac{P_{i}}{P_{A T M}}\right)\right]= \\
& =\frac{\pi \mu \varphi \sqrt{P_{A T M} v_{A T M}} X_{1}^{2}\left(7,418-1,797 X_{1}^{2}\right) t}{24} .
\end{aligned}
$$

Из (12) выразим длительность $t_{C}$ процесса натекания воздуха в межстенную камеру:

$$
t_{C}=\frac{24\left(\left(\frac{P_{i}}{P_{A T M}}\right)_{M A X}-\frac{P_{i}}{P_{A T M}}\right)\left[V+\left(V_{0}-V\right)\left(\left(\frac{P_{i}}{P_{A T M}}\right)_{M A X}+\frac{P_{i}}{P_{A T M}}\right)\right]}{\pi \mu \varphi \sqrt{P_{A T M} v_{A T M}} X_{1}^{2}\left(7,418-1,797 X_{1}^{2}\right)} .
$$

Из выражения (13) видно, что длительность процесса натекания зависит не только от объема межстенной камеры и габаритов системы истечения, но и от динамики перемещения клапана пульсатора. Управление клапаном пульсатора предлагается осуществлять линейным электродвигателем. Применение разработанного пульсатора с управляемыми переходными процессами в межстенных камерах доильных стаканов позволяет получить технологию машинного доения с «щадящим» режимом. При этом длительность фазы с равна 120 мс, что в два раза больше, чем в существующих установках и соответствует физиологическим особенностям процесса молокоотдачи у коров [10]. Усовершенствованный доильный аппарат с разработанным пульсатором уменьшает негативное влияние машинного доения на организм животных, повышает продуктивность коров, снижает возникновение маститов и выбраковку заболевших животных. 


\section{References:}

1. Krasnov IN (1974) Doil'nye apparaty / I. N. Krasnov. - Rostov-na-Donu: Izd-vo Rostovskogo universiteta, 1974. - 228.

2. Krasnov IN (2009) Mehanizacija proizvodstva, pervichnoj obrabotki i pererabotki moloka / I. N. Krasnov. - Rostov-na-Donu: Terra Print, 2009. - 158.

3. Donskoj AS (2009) Matematicheskoe modelirovanie processov $\mathrm{v}$ pnevmaticheskih privodah / A. S. Donskoj. - SPb.: SPbGPU, 2009. - 121.

4. Bolgarskij AV (1975) Termodinamika i teploperedacha / A. V. Bolgarskij, G. A. Muhachev, V. K. Shhukin. - Moscow: Vysshaja shkola, 1975. - 495.

5. Grinchenko VA (2011) Obosnovanie konstruktivno-rezhimnyh parametrov doil'nogo apparata s jelektropul'satorom na osnove linejnogo dvigatelja: dis. ... kand. tehn. nauk: 05.20.01 - Tehnologii i sredstva mehanizacii sel'skogo hozjajstva, 05.20.02 Jelektrotehnologii i jelektrooborudovanie v sel'skom hozjajstve. - Stavropol', 2011. - 197.

6. Grinchenko VA, Laguta II (2014) Problemy mashinnogo doenija i puti ih reshenija // Novye zadachi tehnicheskih nauk i puti ih reshenija. Ufa: Ajeterna, 2014. - pp. 17-18.

7. Grinchenko VA (2009) Ob usovershenstvovanii jelektropul'satora dlja mashinnogo doenija // Tehnika i tehnologija. - 2009. - №1. - pp. 27.

8. (2008) Pat. 79236 Rossijskaja Federacija, MPK8 A $01 \mathrm{~J}$ 5/14. Jelektromagnitnyj pul'sator doil'nogo apparata / Nikitenko G. V., Grinchenko V. A.; zajavitel' i patentoobladatel' Stavrop. gos. agrar. un-t. - № 2008132309/22; zajavl. 05.08.08; opubl. 27.12.08.

9. (2013) Pat. 126563 Rossijskaja Federacija, MPK8 A $01 \mathrm{~J} 5 / 14$. Jelektropul'sator doil'nogo apparata dvojnogo dejstvija / Nikitenko G. V., Kapustin I. V., Grinchenko V. A.; zajavitel' i patentoobladatel' Stavrop. gos. agrar. un-t. - № 2012123542/13; zajavl. 06.06.12; opubl. 10.04.13.

10. Nikitenko GV, Grinchenko VA (2011) Optimizacija rezhima doenija korov // Mehanizacija i jelektrifikacija sel'skogo hozjajstva. - 2011. - №7. - pp. 11-12. 